\title{
Innovación pública: una propuesta de análisis de los factores que inciden en los procesos de innovación en el sector público local
}

\author{
Pilar Mairal Medina ${ }^{1}$
}

Recibido: 15/02/2020 / Aceptado: 21/03/2020

Resumen. La innovación pública es un objeto de investigación emergente que ofrece distintos enfoques para analizar el cambio en las organizaciones del sector público. El ámbito local ha demostrado sus capacidades creativas en este sentido cada vez son más las experiencias de interés relacionadas con el diseño de políticas públicas, el establecimiento de retos y prioridades políticas, y la mejora de los servicios públicos. Sin embargo, y pese a la emergente aparición de espacios no estatales ligados a experiencias innovadoras de participación social, generalmente las administraciones públicas identifican las innovaciones con la integración tecnológica y la transformación cultural de estas organizaciones parece no identificarse con los procesos de innovación.

El artículo resume los principales factores identificados en un estudio empírico que delimitan la percepción de los procesos de innovación pública local y sus resultados, delimitando los condicionantes de estos procesos y los retos que plantean en las organizaciones públicas. Palabras clave: Innovación, gestión pública innovadora, administración local, cultura organizacional, transformación y cambio adaptativo.

\section{[en] Public innovation: key factors affecting the process of innovation in the local public sector}

\begin{abstract}
Public innovation is a target of emerging research that offer different approaches to analyse the change in public sector organizations. Local administrations are showing special creative abilities for innovation. More and more interesting local experiences address new public polices the establishment of political challenges and priorities, and the improvement of public services. However, most frequently local administrations have the tendency to restrict the concept of innovation to the deployment of new electronic systems, machines or industrial devices, neglecting cultural and organizational transformation as real and needed forms of innovation. This limited scope entails a very relevant impact in the innovation success, as even when implementing new technological devices or electronic systems, if the public administration does not pay attention to the needed changes in the organizational and cultural process for implementing such devices, their innovation many times ends up failing.

This paper describes main organizational conditions for a successful process of public innovation, as identified in an empirical research on local administrations. It concludes with some challenges that public administrations will have to face to advance in public innovation.
\end{abstract}

Keywords: Innovative public management; Local administration; Organizational culture, Adaptive change and transformation.

Sumario. 1. Introducción. 2. Metodología de la investigación. 3. El análisis de la innovación en el ámbito local: de las organizaciones públicas a los ecosistemas de innovación. 4. La innovación distribuida y el retorno de valor público en los procesos de innovación local. 5. Los condicionantes de la innovación: factores que operan sobre los procesos de innovación en las administraciones locales. 6. Conclusiones.

Cómo citar: Mairal Medina, P. (2020): Innovación pública: una propuesta de análisis de los factores que inciden en los procesos de innovación en el sector público local, en Cuadernos de Gobierno y Administración Pública 7-1, 53-61.

\section{Introducción}

La innovación pública se ha convertido en un objeto de estudio de creciente interés y también en un foco de atención para el sector público. Mientras que en el ámbito empresarial la innovación se viene planteando como una condición clave en la adaptación de las organizaciones y sus modos de producción a una economía globalizada más competitiva, en el espacio público se plantea como una oportunidad para adaptar la administración y sus servicios a las demandas ciudadanas, con mayores dosis de eficacia, eficiencia y transparencia.

\footnotetext{
Instituto Complutense de Ciencia de la Administración

Universidad Complutense de Madrid

mmairal@cps.ucm.es
} 
Las organizaciones públicas en el entorno local se han convertido durante las tres últimas décadas en un referente claro para la ciudadanía, las organizaciones de la sociedad civil y las empresas, y se han consolidado como colaboradores necesarios de los gobiernos nacionales y regionales en el despliegue de políticas y recursos que se materializan en los territorios. Desde esta perspectiva la transformación del rol de lo local y su emergencia y consolidación como agentes del cambio de paradigma de las políticas públicas ante los retos sociales, económicos, tecnológicos y ambientales, han hecho de estas organizaciones un referente para la innovación, habida cuenta las numerosas experiencias que se vienen desarrollando, la diversidad de sus temáticas y resultados. En esta línea, analizar los factores que inciden en los procesos de innovación pública se plantea como un tema de especial interés para entender qué es innovación pública y diferenciarla de experiencias que, aunque impulsadas por las entidades públicas, no siempre revierten hacia las organizaciones los logros alcanzados.

Para profundizar sobre estas cuestiones del campo de innovación pública en el entorno local, se llevó a cabo un estudio empírico con el objetivo de identificar los principales factores que inciden en los procesos de innovación pública en las administraciones locales. Los siguientes epígrafes recogen los principales resultados de la investigación realizada por la autora.

\section{Metodología de investigación}

La investigación realizada partió de una exhaustiva revisión bibliográfica sobre la que formular un marco conceptual a contrastar empíricamente. Para ello se realizó un análisis exhaustivo de tendencias y prácticas de innovación pública a nivel internacional sobre la se estructuró un marco analítico que fundamentó la investigación empírica, centrada en el análisis de experiencias de innovación pública en el ámbito local español, y en la que se tomó como referencia procesos de innovación basados en nuevos enfoques de política pública que incorporan tendencias internacionales en las áreas de medio ambiente y sostenibilidad, y seguridad ciudadana.

Sobre estas experiencias se realizó un análisis empírico de fuentes documentales y cuantitativas. Para el análisis cuantitativo se elaboró un cuestionario que recogía indicadores de los principales factores de entrada (inputs), procesos y resultados (outputs) del propio proceso de innovación, que habían sido formulados en el marco conceptual inicial.

\section{EI análisis de la innovación en el ámbito local: de las organizaciones públicas a los ecosistemas de innovación}

La innovación en las administraciones públicas se ha analizado por distintos autores y autoras como el resultado de un proceso de cambio en el que se distinguen diferentes orientaciones, como señala Ramírez Alujas (2010). Una clara tendencia ha sido analizar la innova- ción pública como elemento estratégico de la modernización administrativa y de las instituciones para la mejora de la gestión (Borins, 2001; Mulgan y Albury, 2003; Moran, 2004; Osborne y Brown, 2005, 2011; Hartley, 2005; Hartley, Sorensen y Torfing, 2013). Otros estudios se han centrado en el propio proceso de innovación, creando un marco conceptual y analítico que incluye factores y componentes del proceso innovador con el objetivo de generar modelos exitosos de aplicación al sector público (Borins, 2006; Pollitt, 2008; Bason, 2010; Bloch, 2013; OECD, 2016).

La revisión de la literatura existente, tanto en lo referido a los enfoques y las orientaciones gubernamentales de distinto ámbito territorial, como la producción científica y técnica, evidencian una tendencia que en 1981 ya caracterizaron Irwin y Erwin Feller en su artículo "Public Sector Innovation as Conspicuous Production”: la innovación como un objeto de estudio creciente, elevado a la categoría de indicador de rendimiento de actores políticos y administrativos (Feller y Feller, 1981).

El planteamiento del que partimos el estudio realizado descansa en un concepto amplio de innovación pública que hace referencia a los procesos y no solo a los productos, a los enfoques, las metodologías y a los resultados esperados y también a los no esperados y, muy importante, al aprendizaje y conocimiento generado pese a la limitada capacidad y hábito de sistematización y transferencia en las entidades públicas. También al conocimiento surgido del error. En definitiva, a los cambios no disruptivos que han ido configurando nuevos métodos de trabajo en las organizaciones y que, a su vez, han permitido normalizar nuevas visiones de la propia organización y de su impacto sobre el territorio y las personas, respondiendo al modelo de Gestión 2.0 planteado por Ramírez-Alujas en 2010.

La innovación se plantea, por tanto, como un elemento estratégico, debido a que las necesidades y requerimientos de la sociedad son diferentes y evolucionan con gran rapidez en un mundo global e interconectado, en el que gobiernos y organizaciones públicas interactúan con diferentes actores (asumiendo roles diversos y dinámicos) en un escenario de enorme incertidumbre que afecta directamente a lo local.

Los procesos de gestión basados en la gobernanza local (Navarro, 2015) delimitan muchos de los escenarios de innovación pública actual teniendo en cuenta tanto las políticas sectoriales como los ámbitos más generales de gestión pública (Natera, 2004; Longo, 2012; Brugué, Blanco y Boada, 2014; Zurbriggen y Lago, 2014; Mairal, Pastor y García, 2015). En estos escenarios la innovación se plantea como un reto para mejorar la administración desde distintos parámetros institucionales (ajuste presupuestario y reducción de costes, efectividad y eficiencia, o transparencia) aunque también es una oportunidad para consolidar estructuras administrativas con mejores capacidades institucionales para dar respuesta a las demandas ciudadanas.

Desde la perspectiva municipal, el enfoque de la gobernanza local pone de relieve el encuentro de las personas y la ciudadanía con los servicios públicos prestados sobre el territorio y que pretenden dar respuesta a las 
Gráfico 1. Factores que impulsan el entorno local como ecosistema de innovación.

\begin{tabular}{|c|c|c|c|}
\hline Globalización. & $\begin{array}{l}\text { Concentración de la } \\
\text { población en entornos } \\
\text { urbanos. }\end{array}$ & $\begin{array}{l}\text { Desarrollo del municipalismo } \\
\text { y reconocimiento del rol de } \\
\text { las autoridades locales. }\end{array}$ & $\begin{array}{l}\text { Reconocimiento internacional } \\
\text { y europeo de las ciudades y } \\
\text { las políticas urbanas. }\end{array}$ \\
\hline \multicolumn{2}{|c|}{ Gobierno multinivel. } & \multicolumn{2}{|c|}{$\begin{array}{l}\text { Nuevos retos (sociales, medioambientales, } \\
\text { tecnológicos, financieros, sociosanitarios) que } \\
\text { generan nuevos ámbitos y enfoques de política } \\
\text { pública (sostenibilidad ambiental, resiliencia, } \\
\text { transparencia y buen gobierno, etc.). }\end{array}$} \\
\hline $\begin{array}{c}\text { Proactividad en el desarrollo } \\
\text { de espacios colaborativos y } \\
\text { de participación e implicación } \\
\text { ciudadana. }\end{array}$ & $\begin{array}{l}\text { Motivaciones políticas y } \\
\text { técnicas: de la necesidad } \\
\text { de cambio a la oportunidad } \\
\text { de emprender iniciativas, } \\
\text { pasando por las modas } \\
\text { globalizadas. }\end{array}$ & $\begin{array}{c}\text { Reconocimiento y } \\
\text { nuevos planteamientos } \\
\text { para el impulso de redes } \\
\text { colaborativas (intra e inter- } \\
\text { organizativas). }\end{array}$ & $\begin{array}{l}\text { El papel de las alianzas } \\
\text { público-privadas (con ánimo } \\
\text { de lucro o sin él): generación } \\
\text { de conocimiento conjunto, } \\
\text { intercambio de experiencias, } \\
\text { creatividad e inteligencia } \\
\text { colectiva. }\end{array}$ \\
\hline
\end{tabular}

Fuente: (Mairal, 2019).

necesidades más cercanas a su vida cotidiana. Junto a ellos, empresas y organizaciones sociales interactúan en un espacio común que marca la materialización del sistema político-administrativo, de sus objetivos y del rendimiento de su actividad (Longo, 2009). Todo ello en el marco de un escenario cambiante donde los nuevos retos sociales y la proximidad marcan la interacción del gobierno y administración pública con la ciudadanía.

La participación de entidades privadas en la gestión de los servicios públicos viene siendo una realidad creciente, paralela a la transformación de una ciudadanía cada vez más cercana a las administraciones locales, en unos territorios en los que el resultado ha sido un gobierno relacional que vehicula redes de agentes que operan junto a las instituciones públicas, con mayor o menor complejidad y con nuevos instrumentos tecnológicos sobre los que se articulan estas relaciones. Y de estas realidades locales dan buena cuenta las redes de colaboración entre entidades públicas, instituciones privadas con y sin ánimo de lucro y la propia ciudadanía organizada, los equipos intersectoriales, los liderazgos colaborativos, o el desarrollo de gobernanza centrada en la relación con las comunidades locales (Mairal, 2011).

\section{La innovación distribuida y el retorno de valor público en los procesos de innovación local}

Si hay un espacio en el que la innovación se significa en lo local, es el que se vincula a los modelos de gobernanza asociados a procesos colaborativos en el diseño de servicios públicos. Podemos hablar de Gobernanza de la innovación entendida como innovación distribuida (Rivera, Simmonds, y Román, 2012), o innovación en la Gobernanza (Conejero y Redondo, 2016). Estos planteamientos dan cuenta de distintos modelos de cocreación incluyendo a agentes públicos y privados, con distintos formatos de partenariado público-privado para la co-producción de servicios, generando ecosistemas de innovación que confluyen en la iniciativa privada y la apertura de políticas y servicios a nuevos actores.

Los laboratorios de innovación gubernamental (Government Innovation Lab -GIL) son una referencia emergente que se ha generalizado de manera global. Se trata de espacios públicos de innovación (Public Innovation Places -PIP) capaces de afrontar mayores riesgos ante el fracaso que en el marco de estructuras públicas tradicionales y que hacen confluir a organizaciones caracterizadas por una conexión directa con el sector público, creadas para desafiar problemas públicos (Zanardo, 2014). Se trata de un salto en el modelo de co-diseño para abrir a redes de actores los recursos (tangibles e intangibles) públicos y compartir procesos que buscan generar valor público.

En relación a los modelos innovadores de gobernanza (innovación en la gobernanza), estos se centran en mejorar la Gobernanza horizontal, sobre todo en los servicios primarios de atención directa a la ciudadanía, aprovechando en muchos casos las TIC para mejorar las capacidades de comunicación. Uno de los primeros ejemplos en Europa fue el lanzamiento en 2009 de Total Place en el Reino Unido, un programa centrado en las administraciones locales para aprovechar al máximo el trabajo conjunto de recursos y servicios promoviendo nuevas formas de cooperación a nivel local, y entre el nivel local y la administración central ${ }^{2}$.

Este tipo de programas evidenciaron la necesidad de incluir enfoques más integrados en la gestión con ser-

Partiendo de 13 proyectos pilotos se buscó establecer estrategias de mejora de servicios públicos en materia de empleo, prevención de drogodependencias, tratamiento de personas en riesgo y seguridad ciudadana. El papel del tejido asociativo, junto a colectivos empresariales y la propia ciudadanía, puso de manifiesto el papel de los servidores públicos en el diseño y la supervisión de iniciativas innovadoras mejorando los procesos de evaluación (Office of Public Sector Information, 2010). El aspecto clave del modelo Total Place fue poner en práctica un concepto holístico de los servicios públicos a través de unidades abiertas para el co-diseño y la gestión coordinada de servicios en los territorios. 
vicios centrados en el ciudadano en vez de mantener la lógica tradicional de los servicios sectoriales en mayor o menor medida aislados en sus procesos de planificación, gestión y evaluación (salud, seguridad, servicios sociales, etc.). La replicabilidad de estos modelos (transversalidad y coordinación del gobierno multinivel) inicialmente ofrece distintas oportunidades, pese a que requiere nuevos modelos de coordinación y gestión de la información, habitualmente dificultosos en estructuras que gestionan servicios excesivamente departamentalizados, en silos, y que en ocasiones alimentan lógicas "not my job" (Lee y Olshfski, 2002).

Como observamos, las iniciativas de innovación pública local se centran frecuentemente en las estructuras y procesos de gestión de los recursos. El énfasis en la participación y colaboración de todos los actores dentro de un territorio se ha desplegado también desde el nivel más micro, al barrio o distrito con enfoques comunitarios. Se trata de abordar los problemas poliédricos desde soluciones colectivas y multidimensionales. Ejemplos de esta tendencia son programas como los ya consolidados en Berlín (Quarter Management) o el despliegue de los Buurt Managers en varios municipios de Holanda. Se basan en sistemas de gestión comunitaria, que pueden incluir equipos inter-sectoriales, redes público-privadas y consejos ciudadanos, coordinadas para la implementación de actuaciones sinérgicas que abordan los problemas del territorio de forma sistémica, y ponen el valor la inteligencia colectiva (Klok, Denters, y Vrielink, 2013). Ambos casos parten de un enfoque de rediseño organizativo y distribución de funciones entre actores públicos y privados.

Como se ha apuntado ya, en la actualidad es difícil encontrar un proceso de innovación pública en el que no se subraye la importancia de la participación ciudadana o de la población usuaria de los servicios. Distintas metodologías de co-producción se han implementado en muchas localidades de la Unión Europea (Schaap, Geurtz, De Graaf y Karsten, 2010). En este punto el concepto de innovación distribuida pone el foco en el papel activo del ciudadano en la co-producción y muchas veces la co-gestión y auditoría de los servicios. La utilización de soluciones digitales de nuevo ha impulsado formatos de participación ciudadana más difusos y ofrecido nuevos espacios de interacción con el gobierno en el proceso decisional (la normalización de plataformas tecnológicas y aplicaciones generadas para recoger propuestas, realizar consultas ciudadanas o realizar votaciones electrónicas en el marco de los presupuestos participativos da buena cuenta de ello), pese a las limitaciones que plantea la brecha tecnológica. En todo caso, estas tendencias se consolidan en el tiempo sentando las bases de futuros enfoques y metodologías de gestión que serán alimentadas por nuevas soluciones tecnológicas.

De igual forma también desarrollado experiencias diversas para facilitar el uso de crowd sourcing a través de APIS (Application Programming Interface) que permiten la contribución con ideas y conocimientos, abiertas a cualquier ciudadano, y la elaboración colectiva de planes o proyectos, así como la canalización de recursos monetizados. Ejemplos novedosos durante la primera década de este siglo fueron el programa "Cabinet Office's Tell Us how" (Reino Unido), el uso de cartografía participativa sobre problemas o información diversa, como Geodata en Alemania, o los mapas de delitos en EEUU y Reino Unido. También la iniciativa Fixyourstreet en Irlanda, para comunicar incidencias en el territorio sobre una base cartográfica que se viene alimentando desde 2011 para informar sobre situaciones que requieren la acción pública y sobre las cuales la ciudadanía obtenía una respuesta directa por parte de los Consejos locales (Rivera, Simmonds, y Román, 2012). A partir de estos casos de referencia desde la primera década de siglo, las experiencias han sido múltiples y los formatos y ámbitos de política pública diversos y como se avanzaba anteriormente, las oportunidades son diversas atendiendo al acelerado ritmo de la evolución y la transformación digital.

En esta línea, las experiencias que se orientan hacia el gobierno abierto son numerosas y variadas y en la actualidad se siguen desarrollando múltiples iniciativas que, sin duda, serán objeto de investigación social desde distintas perspectivas disciplinares. Una de las líneas más interesantes se alimenta de herramientas para facilitar el uso de datos abiertos ofrecidos por las administraciones públicas para su reutilización. En este ámbito, el avance es vertiginoso en la actualidad cada vez son más y mejores (en términos de implementación y de amplitud de datos) las herramientas desarrolladas por el sector público local para apoyar el desarrollo de soluciones que entran en el mercado para ofrecer servicios digitales relacionados con el territorio, y que son puestas a disposición de la ciudadanía. En este punto, conviene resaltar un modelo que evidencia la aportación de la acción pública y la información generada al desarrollo de nuevos mercados en la economía digital: las administraciones ponen a disposición conjuntos de datos abiertos (catálogos) que desarrolladores privados utilizan para alimentar aplicaciones que prestan servicios diversos, y que son accesibles a la ciudadanía para realizar consultas, enriqueciendo estos catálogos con nuevos datos. La información se publica regularmente (datos abiertos) y de forma reutilizable para todo el mundo, sin restricciones de acceso, patentes $\mathrm{u}$ otros mecanismos de control o monetización, más allá de los parámetros que delimitan los marcos regulatorios de la protección de datos personales.

Sin embargo, cabe plantearse: pese a que estos modelos de innovación en entornos de gobernanza avanzan principalmente desde la perspectiva de la participación ciudadana, los servicios digitales y las redes de política, ¿se motiva a las estructuras organizativas a participar o a alinearse con los procesos de participación, más allá de entender esta participación como un servicio y no como una herramienta? ¿Se han institucionalizado métodos de trabajo inter-institucionales efectivos, que garanticen un rendimiento óptimo en escenarios de coordinación intergubernamental o inter-organizativos?

Como hemos señalado, mientras que durante la última década ha emergido un interesante espacio de oportunidad para el análisis de la innovación pública en el ámbito local, las publicaciones científicas muestran un especial interés por abordar procesos de participación en la coproducción de servicios más allá de analizar las innova- 
ciones que han llevado al incremento de buenas prácticas en esta materia (Newman, Raine, y Skelcher, 2001; Pastor, 2018) y sus retornos hacia las organizaciones públicas. De facto, recientemente se observa un incremento en la visibilización de experiencias ligadas a la innovación social que en ocasiones es equívocamente tratada como innovación pública, pese a que estas experiencias pongan el foco en el desarrollo de iniciativas de carácter comunitario, inter-emprendedor, etc., y se enmarquen con claridad en espacios públicos no estatales (Bresser-Pereira y Cunill, 1999). Se trata en muchos casos de iniciativas apoyadas por gobiernos y administraciones locales que, como plantea Carles Ramió, crean valor público "trabajando en red, aunque dominando la red" (Ramió 2009: 20), pese a que no siempre sus rendimientos o utilidades revierten en las propias organizaciones públicas.

En este punto, podríamos plantear también qué motivaciones de partida impulsan a las administraciones locales a innovar. ¿Son conscientes de las innovaciones que ya han desarrollado? ¿Requieren nuevos recursos o disponen de los suficientes pese a que no cuenten con estrategias y metodologías para afrontar procesos de innovación de manera consciente y planificada? ¿Quién asume los riesgos de innovar? Sin duda algunos de los planteamientos anteriores podrían realizarse sobre organizaciones públicas del ámbito nacional o regional, aunque la complejidad del ámbito local (competencial, financiero, organizativo, relacional) permite ofrecer una visión pormenorizada de factores que intervienen en los procesos de innovación, su relación e impacto sobre las propias organizaciones.

\section{Los condicionantes de la innovación: factores que operan sobre los procesos de innovación en las administraciones locales}

Más allá de los métodos de trabajo aplicados a los procesos de innovación, especialmente ligados en la actualidad a procesos de co-producción de políticas y servicios y enmarcados en experiencias de innovación social, se pueden establecer elementos propios que caracterizan la innovación pública en el ámbito de la administración local.

Estos procesos se han basado en la búsqueda de una mayor eficiencia de los recursos administrativos y han puesto el foco en la integración digital para impulsar la modernización administrativa a través de la administración digital, en una primera fase, a la que ha seguido el despliegue de líneas de innovación que se asocian a distintos ámbitos de política pública (salud, servicios basados en el enfoque de Smart City para la telegestión de recursos y suministros básicos, promoción y dinamización económica, participación ciudadana, etc.). Estas innovaciones están avanzando con la integración de nuevas soluciones basadas en el big data y la inteligencia artificial para proveer nuevos servicios $y$, desde la perspectiva de las funciones core del sector público, permitirán incorporar nuevas herramientas de planificación, interacción y seguimiento de los recursos públicos.

Igualmente, el avance en materia de participación ciudadana ha supuesto la normalización de herramientas orientadas hacia la mejora de los canales y métodos de interacción entre las administraciones locales con la ciudadanía, y en la mayoría de los casos el foco se ha puesto en digitalizar procesos de consulta y también de comunicación con la población (servicios de atención e información, teleasistencia, presupuestos participativos, transparencia).

En este punto, y desde una perspectiva amplia, conviene resaltar el impacto de distintos y diversos procesos de colaboración público-privada en el avance de los procesos de innovación en el ámbito público local. Y es que, tanto la interacción con proveedores privados como la colaboración con el tercer sector, centros tecnológicos o equipos de investigación, han supuesto el reconocimiento institucional y desarrollo de las capacidades de los entornos locales para impulsar ecosistemas de innovación. Una vez más, se reconoce en estos entornos de generación de nuevas capacidades compartidas modelos de gobernanza que ponen de relieve nuevos métodos de coordinación, sobre los que se alinean objetivos comunes y se comparten experiencias y recursos.

A pesar de ello también se localizan retos clave vinculados a factores relacionados con el cuestionamiento de las capacidades políticas de la propia administración local, tales como la movilización sostenible de la ciudadanía y el salto, de nuevo, desde el co-diseño a la co-producción o, desde otra perspectiva, desde la participación a la implicación de la población, y del propio personal técnico, en los propios procesos de innovación pública y también en la propia implementación de las políticas y servicios generados.

Desde esta perspectiva, además de la motivación ciudadana y de los múltiples factores sociopolíticos que inciden sobre su voluntad y actitud hacia la participación activa, se debe destacar el excesivo peso de los medios digitales en estos procesos y las limitaciones que las soluciones aplicadas tienen en organizaciones públicas que no han hecho frente a la transformación digital por factores culturales o financieros, así como las disfuncionalidades asociadas a la brecha digital.

Otro de los retos localizados asociados a los procesos de innovación son los retornos de valor público. Se entiende que la diversidad de agentes y stakeholders, los modelos de gobernanza aplicados y la confluencia de distintos recursos, pueden generar efectos multiplicadores en las capacidades de innovación pública local. Sin embargo, los retornos a las organizaciones del sector público no siempre son tangibles y, por ende, reconocidos y dimensionados. Y en este término, la sistematización de resultados y aprendizajes interviene como un factor clave asociado también al reconocimiento institucional, la adaptación organizacional y la permeabilidad y sostenibilidad de los cambios generados, ya sea desde el punto de vista organizativo, metodológico o tecnológico.

Desde el punto de vista de los factores que inciden en los procesos de innovación, los resultados de la investigación realizada indican la significativa relevancia del liderazgo e implicación de los gestores políticos y técnicos durante el proceso de innovación, el conocimiento y capacitación de la estructura municipal en materia de innovación de procesos, las formas de implicación de los trabajadores, y los incentivos para la innovación. 
Figura 1. Características más comunes de la innovación en la gestión pública local.

\begin{tabular}{|c|c|c|}
\hline MOTORES DE INNOVACIÓN & LÍNEAS DE INNOVACIÓN & RETOS \\
\hline $\begin{array}{l}\text { La eficiencia en la gestión de los } \\
\text { recursos. }\end{array}$ & $\begin{array}{l}\text { Los servicios públicos digitales y la } \\
\text { administración electrónica. }\end{array}$ & $\begin{array}{l}\text { La incertidumbre política y las reticencias ante } \\
\text { la implicación ciudadana. }\end{array}$ \\
\hline $\begin{array}{l}\text { El acceso a co-financiación. } \\
\text { El desarrollo de soluciones digitales y }\end{array}$ & $\begin{array}{l}\text { La integración de servicios de } \\
\text { Smart City. }\end{array}$ & $\begin{array}{c}\text { Dificultades para conseguir la participación } \\
\text { de los colectivos más vulnerables y la brecha } \\
\text { digital. }\end{array}$ \\
\hline $\begin{array}{l}\text { La demanda ciudadana de espacios de } \\
\text { participación. } \\
\text { La colaboración público-privada. } \\
\text { La competitividad entre territorios. }\end{array}$ & $\begin{array}{c}\text { distribuida. } \\
\text { Las estructuras participativas } \\
\text { de gestión de los recursos de la } \\
\text { comunidad local (co-producción, } \\
\text { co-gestión). }\end{array}$ & $\begin{array}{l}\text { El excesivo peso en la tecnología y no en los } \\
\text { procesos, la adaptación de las organizaciones } \\
\text { y sus estructuras, así como en el rediseño, } \\
\text { testeo y evaluación de nuevas metodologías de } \\
\text { coordinación y gestión de la información. }\end{array}$ \\
\hline & $\begin{array}{l}\text { La comunicación digital con } \\
\text { la ciudadanía (transparencia, } \\
\text { interacción) }\end{array}$ & $\begin{array}{l}\text { La rendición de cuentas. } \\
\text { La captura de los recursos generados en el } \\
\text { marco de iniciativas que buscan incrementar el } \\
\text { valor público de la acción institucional. }\end{array}$ \\
\hline
\end{tabular}

Fuente: Elaboración propia.

De partida, se pueden establecer varios factores que son considerados como inductores en el impulso de iniciativas de innovación pública. El primero de ellos, el liderazgo político, se entiende como un motor para la puesta en marcha de los procesos de innovación y se vincula a la motivación y la canalización de información en las organizaciones en términos de establecer objetivos, y desde este punto de vista se puede establecer como un elemento inspirador, a la par que instrumental, de la acción de gobierno y un indicador de rendimiento al mejorar el análisis de viabilidad de los procesos en las organizaciones.

Este input se vincula a otro que opera en un doble sentido. Se trata de la toma de decisiones para encontrar alterativas ante las reducciones presupuestarias llevadas a cabo tras la radical caída de ingresos del sector público local a raíz de la crisis global de 2008. En el sentido contrario, la falta de recursos presupuestarios se percibe también como un freno a las capacidades de las organizaciones para potenciar procesos de cambio y se establece la necesidad de contar con recursos adicionales para impulsar la innovación pública, bien sea a través de más recursos humanos, rúbricas disponibles para contar con servicios externos o ejecutar inversiones. En este contexto la participación de las entidades públicas locales en fondos $\mathrm{y}$ ayudas que permiten co-financiar proyectos de innovación, se ha demostrado un acicate para potenciar procesos de cambio y mejora basados en soluciones y métodos ya existentes, testados en otros entornos locales o regionales.

Lo anterior avanza otro de los factores de partida que se perciben como claves, como es el grado de capacitación de las estructuras técnicas para abordar estos procesos en términos de manejar herramientas y técnicas de nueva implantación, conocer las lógicas de los proyectos para poder gestionar el seguimiento o la comunicación de los objetivos logrados, o también conocer experiencias similares desarrolladas en otros contextos para anticiparse a los desafíos que suponen estos procesos para las propias organizaciones (cambios en las estructuras y metodologías de trabajo, nuevas soluciones tecnológicas que requieren de competencias específicas, etc.).

En el propio proceso de implantación de las innovaciones también se han detectado factores que operan como impulsores y motivantes o, en el sentido contrario, como barreras que obstaculizan el desarrollo de estas innovaciones. Como veremos a continuación, los elementos detectados se pueden analizar agrupándolos en cuatro dimensiones: la dimensión política, la cultura organizacional, los factores metodológicos e instrumentales y la dimensión de gobernanza.

El primero de estos condicionantes es el compromiso político en términos de liderazgo e implicación de la organización para alinearla con los objetivos de innovación. La innovación como proyecto político, da cuenta de un factor de gran relevancia desde el inicio para movilizar recursos y motivar e implicar a las estructuras técnicas en un proyecto viable con capacidad para ser sostenible en el tiempo por su lógica de respuesta a las necesidades de la organización y su entorno.

Se asocian a este factor la percepción de falta de incentivos para la innovación en los equipos y el interés y la motivación del personal municipal, a su vez claves en la normalización de actitudes favorables al cambio. Se trata de condicionantes vinculados a la cultura organizacional y a las dinámicas propias de las organizaciones del sector público que, en el caso de las administraciones locales, conjugan singularidades relacionadas con la visualización de los impactos sobre el territorio, o la diversidad de los ámbitos de política pública en los que interactúan los equipos técnicos. También con los impactos de políticas de contracción del gasto sobre sus estructuras y también sobre las políticas y servicios que gestionan, pese a que los marcos de política pública local se han ampliado con nuevos enfoques que responden a los retos sociales y ambientales de carácter global a los que deben dar respuesta. 
Por su parte, y como se ha avanzado anteriormente, también se puede plantear una dimensión metodológica que aglutina factores clave de proceso. Destacan en este punto el diseño de sistemas de supervisión y seguimiento asociados al proceso de innovación en cada una de sus fases para valorar los resultados obtenidos, analizar potenciales cuellos de botella y tomar decisiones de ajuste y mejora desde un punto de vista técnico. También el grado de conocimiento de metodologías de innovación es un factor clave para alinear los objetivos y esfuerzos de las estructuras técnicas con el proceso y sus lógicas, y la identificación de estos procesos con la innovación. Se percibe en este sentido que no siempre se visualizan como resultado de la innovación los cambios generados, o las nuevas herramientas implementadas, y esta circunstancia minimiza los retornos a la organización en términos de motivación hacia nuevos procesos.

Finalmente, y relacionado con la creciente implementación de soluciones digitales proveedoras de servicios internos y externos en las organizaciones públicas, se percibe que el grado de integración de herramientas digitales, es otro de los factores instrumentales clave del propio proceso de innovación.

La cuarta dimensión sobre la que se articulan los factores de proceso está relacionada con la interacción de los agentes que componen las organizaciones públicas locales, tanto desde un punto de vista técnico como político, con otros agentes de su entorno. El enfoque de gobernanza permite establecer factores asociados a estas dinámicas. El primero es de carácter interno y se relaciona con la gobernanza en las propias organizaciones y la visión de la multidisciplinariedad y la coordinación inter-áreas en la gestión de los procesos de innovación. Se trata de un factor percibido como relevante para alcanzar éxitos y que la innovación sea permeable en las organizaciones y el cambio se valore como un avance en sentido positivo, minimizando las resistencias y potenciando las capacidades de las estructuras de trabajo.

Esta visión se corresponde con la tesis de partida que pone de relieve la incorporación de nuevos enfoques de política pública, más integrados y transversales, en las organizaciones locales, principalmente por la necesidad de adaptarse al cambio social, económico y tecnológico para dar respuesta a los retos que el entorno plantea y que en la mayoría de los casos requiere de enfoques más integrados para lograr impactos más certeros. La transformación digital necesaria para implementar la administración electrónica a través de soluciones más o menos integradas, la multidimensionalidad del cambio climático, la apuesta por la sostenibilidad ambiental (eficiencia de consumos, información y sensibilización, resiliencia urbana, etc.), la lucha contra la pobreza (promoción del empleo y el autoempleo, atención a colectivos en riesgo de exclusión social), o las crisis sanitarias como las que evidencian pandemias globales como el COVID-19, son claros ejemplos de la emergencia de modelos más transversales y colaborativos de gestión de servicios locales.

En paralelo, tanto la generación de alianzas con stakeholders facilitadores de los procesos de innovación pública, como la búsqueda de apoyos externos para acceder a recursos técnicos y tecnológicos, se vinculan con distintos modelos de colaboración público-privada, más allá de los marcos contractuales establecidos para materializar esta interacción. La suma de recursos y, especialmente de conocimientos y visiones sobre los procesos de innovación, se percibe como una oportunidad relevante por parte de las estructuras técnicas. Esta percepción, fundamentada en una visión de la innovación y las capacidades organizativas del sector público claramente alineadas con las limitaciones que ya planteaba el enfoque de Nueva Gestión Pública, hace que re-emerjan demandas de colaboración con agentes para la transferencia de metodologías propias del sector empresarial (marcos LEAN y metodologías ágiles) a procesos de innovación incremental en las organizaciones públicas locales.

Por su parte, la generación de redes colaborativas con el tercer sector implicando a comunidades en procesos de co-diseño de políticas e incluso la co-producción de servicios de proximidad, se enmarcan también en esta dimensión de gobernanza.

Figura 2. Factores que inciden en los procesos de innovación pública local.

\section{FACTORES DE PARTIDA}

\section{Liderazgo político.}

Reducción presupuestaria.

Grado de implicación inter-áreas en procesos de innovación.

Capacitación del personal técnico.

Percepción de viabilidad de los procesos (diseño técnico basado en necesidades y metodologías de coordinación del proceso).

\section{FACTORES DE PROCESO}

Compromiso e interés político (la innovación como proyecto político)

Percepción de falta de incentivos para la innovación. Interés y motivación del personal municipal.

Las actitudes favorables hacia el cambio.

Existencia un sistema de supervisión y seguimiento.

Grado de conocimiento sobre las iniciativas de innovación y su identificación como experiencia innovadora.

Grado de integración digital.

Necesidad de apoyo de profesionales externos.

Participación de equipos multidisciplinares y multisectoriales. 


\section{Conclusiones}

En este artículo se recogen algunos elementos concluyentes en torno a la innovación pública local. El panorama general muestra un extenso catálogo de experiencias en pocos casos basadas en la evidencia o en procedimientos sistematizados de implementación que favorezcan evaluaciones rigurosas o estudios comparados. Sin embargo, el reto de abordar los factores que condicionan la innovación pública local, permite establecer a las organizaciones públicas como agentes de cambio adaptativo y a sus cuadros técnicos, potenciales prescripciones de la innovación.

Con la reflexión realizada se pretenden sintetizar algunos de los aspectos que aportan propuestas de análisis en torno a la innovación pública y el rol emergente de los gobiernos y administraciones locales en esta materia. Sin duda, estos aspectos requieren de una mirada multidimensional, integradora y pluridisciplinar para abordar los retos que plantea un objeto de estudio complejo (Mairal, 2018).

Como se ha señalado al inicio, en contraposición a lo que sucede en la esfera privada, la innovación no se ha planteado tradicionalmente en las administraciones públicas como un condicionante para la transformación y la mejora de la acción pública. Las razones pueden encontrarse en la falta de incentivos y motivaciones, y el elevado riesgo que se asocia a la innovación por la incertidumbre que plantea en relación a los resultados, su impacto político o su rendimiento técnico, a la necesidad percibida de invertir recursos extraordinarios, etc. (ANAO, 2009; Arundel, Bloch y Ferguson, 2019).

Pese a lo anterior sí se ha consolidado un relato teórico en relación a la relevancia que el papel de la innovación ha tenido históricamente en el sector público como motor de mejora en la eficacia y eficiencia de los servicios públicos. No obstante la percepción de la innovación es un factor clave para entender la capacidad de las propias organizaciones públicas para innovar y se pueden establecer determinadas limitaciones relacionadas con la identificación del impacto del proceso y los resultados de las innovaciones locales desarrolladas (adaptaciones no disruptivas de las organizaciones, mejoras en procesos de coordinación, incorporación de nuevos enfoques integrados como marcos referenciales de las políticas locales y los marcos metodológicos en las organizaciones, etc.). En este sentido, se puede avanzar que en muchos casos la mejora en los procesos internos, el rendimiento administrativo y financiero, en términos de agilidad, eficacia o ahorro de costes, no siempre son valorados cuando la evidencia de los resultados no es inmediata (Mairal, 2018).

La investigación permite concluir que el éxito o fracaso de una innovación pública no solo dependerá de las cualidades propias del proyecto innovador, sino también de una serie de características de la organización y de su contexto que afectan al proceso de desarrollo de la innovación y que son gestionadas de manera más o menos exitosa.

En relación a los factores determinantes, la investigación realizada permite concluir que existen factores que inciden directamente en las propias organizaciones como condicionantes de partida, que pueden ser planteados desde un enfoque sistémico de los procesos de innovación. De igual forma, los propios procesos incidirán sobre los resultados de la innovación.

Podemos por tanto plantear que los elementos organizacionales son claves para analizar cómo se despliegan los procesos de innovación pública y cuáles son los puntos calientes para abordar la planificación: la capacitación, la flexibilidad, las limitaciones en la coordinación y la comunicación inter-áreas, motivación, identificación de la innovación y sus resultados... son factores que inciden directamente en las capacidades innovadoras de las organizaciones públicas y en lo local, se plantean como condicionantes de los espacios de interacción con otros agentes.

Probablemente estos inputs sean los que motiven el esfuerzo por concentrar las innovaciones locales en iniciativas sectoriales articuladas a modo de proyectos de base tecnológica y en escenarios de menor riesgo institucional. En definitiva, réplicas de soluciones que no consideran la escalabilidad y que se asientan sobre procesos de testado y experiencias de desarrollo previos en otros entornos.

A modo de conclusión se pueden también plantear algunos de los desafíos observados tales como la revisión de las arquitecturas organizativas, el salto (en los procesos de innovación) desde el co-diseño hacia la implementación de soluciones compartidas y la escalabilidad, el liderazgo sostenible de las organizaciones públicas y la permeabilidad hacia los retornos de la innovación (conocimiento, experiencia, metodologías), o la propia sostenibilidad de la participación de agentes no estatales en los procesos, facilitando la implicación a medio y largo plazo, y fortaleciendo la co-responsabilidad.

\section{Bibliografía}

Arundel, A., Bloch, C. y Ferguson, B. (2019). “Advancing innovation in the public sector: Aligning innovation measurement with policy goals”, en Research Policy, vol. 48, n 3, pp.789-798. DOI: https://doi.org/10.1016/j.respol.2018.12.001

Australian National Audit Office - ANAO (2009). Innovation in the Public Sector: Enabling Better Performance, Driving New Directions. Best Practices Guide, Commonwealth of Australia.

Bason, C. (2010). Leading public sector innovation: Co-creating for a better society, Bristol: Policy Press.

Bloch, C. y Bugge, M. M. (2013). "Public sector innovation: From theory to measurement", en Structural change and economic dynamics, $\mathrm{n}^{\circ} 27$, pp. 133-145. DOI: https://doi.org/10.1016/j.strueco.2013.06.008

Borins, S. (2001). "Encouraging innovation in the public sector", en Journal of intellectual capital, vol. 2, n 3, pp. $310-319$. DOI: https://doi.org/10.1108/14691930110400128 
Bresser-Pereira, L. C., y Cunill, N. (1999). "Entre o Estado e o mercado: o público não-estatal”, en Bresser-Pereira, L. C., y Cunill, N., O público não-estatal na reforma do Estado. Rio de Janeiro: FGV, pp. 15-48.

Brugué, Q, Blanco, I. y Boada, J. (2014). “Entornos y motores para la innovación en las políticas públicas”, en Revista del CLAD Reforma y Democracia, n59, pp. 5-34.

Conejero, E., y Redondo, J. C. (2016). "La innovación social desde el ámbito público: Conceptos, experiencias y obstáculos”, en Gestión y análisis de políticas públicas, nº 15, pp. 23-42. DOI: https://doi.org/10.24965/gapp.v0i15.10310

Feller, I., \& Feller, E. (1981). "Public-Sector Innovation as Conspicuous Production", en Policy Analysis, vol. 7, nº1, pp. 1-20.

Hartley, J. (2005). "Innovation in governance and public services: Past and present". Public money and management, vol. 25, $\mathrm{n}^{\circ}$ 1, pp. 27-34. DOI: https://doi.org/10.1111/j.1467-9302.2005.00447.x

Hartley, J., Sorensen, E. y Torfing, J. (2013). "Collaborative innovation: A viable alternative to market competition and organizational entrepreneurship”, en Public Administration Review, vol. 73, nº 6, pp. 821-830. DOI: https://doi.org/10.1111/ puar. 12136

Lee, S. y Olshfski, D. (2002). “Employee commitment and firefighters: It's my job”, en Public Administration Review, nº 62, pp. 108-114. DOI: https://doi.org/10.1111/1540-6210.62.s1.19

Longo, F. (2012). "Innovación en Gobernanza: entornos colaborativos y liderazgos relacionales", en EKONOMIAZ, vol. 80, n 02, pp. 46-69.

Mairal, P. (2011). "Incorporando procesos innovadores en la gestión del territorio. Modelos y casos de colaboración públicoprivada en el diseño de políticas y la gestión de servicios de proximidad”, en ponencia presentada en el X Congreso AECPA. Murcia, septiembre.

Mairal, P. (2018). "Gestión pública innovadora en las administraciones locales", en ponencia presentada en el II Congreso Internacional del Instituto Complutense de Ciencia de la Administración. Madrid, noviembre.

Mairal, P. (2019). "La innovación pública en las Administraciones locales". Tesis doctoral. Madrid: Universidad Complutense de Madrid.

Mairal, P., Pastor, G., y García, M.J. (2015). “La gobernanza local: Retos y oportunidades”, en Gandarillas M. y Cueva A. (Coord.). La gobernanza de la seguridad y justicia en la sociedad diversa. Madrid: Ayuntamiento de Arganda del Rey, pp. 25-40.

Mulgan, G. (2014). Innovation in the public sector. How can public organisations better create, improve and adapt? Londres: Nesta.

Mulgan, G. y Albury, D. (2003). Innovation in the public sector. Strategy unit. London: Cabinet Office, pp. 1-40.

Natera, F. (2004). "La noción de gobernanza como gestión pública participativa y reticular", en Documentos de Trabajo Política $y$ Gestión, $\mathrm{n}^{\mathrm{o}} 2$, pp. 1-35.

Navarro, C. J. (2015). “Innovación social y Gobernanza urbana”, en Subirats, J. y García Bernardos, A. (ed.). Innovación Social y Politicas Urbanas en España. Barcelona: Icaria, pp. 43-57.

Newman, J., Raine, J. y Skelcher, C. (2001). “Developments: Transforming local government: Innovation and modernization”, en Public Money and Management, vol. 21, n 2, pp. 61-68. DOI: https://doi.org/10.1111/1467-9302.00262

OCDE (2016). Innovation in the public sector: Making it happen. Observatory of Public Sector Innovation (OPSI).

Osborne, S. P. y Brown, K. (2005). Managing change and innovation in public sector organisations. London/New York: Routledge.

Osborne, S. P. y Brown, L. (2011). "Innovation in public services: Engaging with risk”, en Public Money \& Management, vol. 31 , $\mathrm{n}^{\mathrm{o}}$ 1, pp. 4-6. DOI: https://doi.org/10.1080/09540962.2011.545532

Pastor, G. (2018). "Colaboración abierta e innovación pública”, en Rodríguez, J. y Lariguet, G. Gobierno abierto y ética. Argentina: Universidad Nacional de Córdoba, pp. 239-272.

Pollitt, C. (2008). La innovación en el sector público. Discurso de Apertura de la Conferencia Anual del Grupo Europeo de Administración Pública. Erasmus Universiteit Rótterdam. Holanda.

Ramió, C. (2009). La colaboración público-privada y la creación de valor público. Barcelona: Diputación de Barcelona.

Ramírez-Alujas, A. (2010) "Innovación en la Gestión Pública y Open Government (Gobierno Abierto): Una Vieja Nueva Idea", en Revista Buen Gobierno, no 9. DOI: https://doi.org/10.35247/buengob_09_06

Rivera, L., Simmonds, P. y Román, L. (2012). Trends and challenges in public sector innovation in Europe. Bruselas: DG Enterprise, Comisión Europea.

Zanardo, S. (2014). "EU support for cities and social innovation. SEISMIC project Societal Engagement", Science, Mutual learning In Cities. Policy watch, 1. Bruxelas: Eurocities.

Zurbriggen, C. y Lago, M. G. (2014). "Innovación y co-creación. Nuevos desafíos para las políticas públicas”, en Revista de Gestión Pública, vol. 3, nº 2, pp. 329-361. 